\title{
SURVEY THE OPINION AND INTEREST OF STUDENTS-PEDAGOGUES TO THE SPORT ANIMATION PRACTICE IN AN EDUCATIONAL ENVIRONMENT
}

\author{
D. Zheleva-Terzieva*
}

Faculty of Education, Trakia University, Stara Zagora, Bulgaria

\begin{abstract}
The modern, dynamic and high-tech world sets new challenges for the man as a social and biological being. Lowering of the motor activity of people is a general phenomenon, leading to a number of adverse health and social problems, especially among the younger generation.

This article introduces the problem with insufficient amount of motor activity, realized in the teaching forms on physical education at school age, and studies the interests of students-educators to the subject "Sport animation in the educational environment" in search of additional forms of sports activities for ignoring hypodinamics and its consequences.
\end{abstract}

Key words: reduced motor activity, vocational-pedagogic competence, students of pedagogical specialties, attitudes to sport animation competence.

\section{INTRODUCTION}

The level of technological advancement in today's dynamic world has made life considerably easier. However, freeing people from doing physical work, combined with the unhealthy living conditions - long stay in front of the computer and the TV screen, the systematic use of the means of transport, as well as a number of technical facilities used in everyday life, favour the limiting of the person's motor activity. Hypodinamics nowadays is one of the main reasons for a lot of major diseases. By reviewing the data in existing literary sources, it can be summarized that there is no structure or function in the human body that is not affected by this social disaster, causing severe and irrevocable effects on the physical and the functional abilities of individuals.

The world and European positions firmly promote physical culture as an important value system of life in modern society. The programme of the World Health Organization "HEALTH 2020" [1] provides for the compulsory development of active motor

\footnotetext{
*Correspondence to: Donka Zheleva-Terzieva, Armeiska str, 9, Faculty of Education, Trakia University, Stara Zagora, Bulgaria, Mobile Phone: +359888089871,donkaterzieva2006@yahoo.com
}

regime of life for every person, especially for children and young people, and this is stated as a priority health-care task that should be realized from an early childhood. It is no surprise that "The right of children to move every day" is protected by the Convention on the Rights of the Child [2], because it is the movement that stimulates the growth and development of the growing organism. Active physical activity develops and improves motor skills, the body becomes proportionate, a higher level of efficiency and adaptability to the surrounding environment is reached, developed is a higher level of readiness for dealing with different types of activities, including, crisis and extreme situations.

The progress of the evolutionary development of man predetermines the normal functioning of all organs and systems of the organism in the conditions of active motor activity. Therefore, the planned use of physical exercises and sport in the regime of work and rest becomes a means of preventing the adverse consequences of hypodinamics. To preserve the good health of children, the World Health Organization recommends as a minimum volume 60 and more minutes, 5 times a week of exercise and sport activities [1]. 
The data from a representative study conducted [3] with students aged 6-19 in the period 20102011 shows that the recommended physical activity is achieved only by $24 \%$ of surveyed individuals, and $35 \%$ of the children are physically active less than two days a week.

It was found In a number of studies and publications $[3,4,5]$, that the reduced motor activity in adverse economic status and complex effects of irrational and unbalanced diets, harmful habits and habitudes such as smoking, drinking, drug habits, environmentally polluted environment and others, lead to increased morbidity from an early age, retardation of capacity, unsatisfactory immune boosting and low resistance to unfavourable conditions.

More and more, the prevailing opinion is that there is a need for pre-defining of forms of work in physical education corresponding to the new requirements of the dynamic global economy and to the challenges faced by people in the new technological century [6]. Given the regulated time for a lesson work, for which unfortunately, there are no prospects to be increased soon, the reserves to increase the physical activity of students should be sought in the extracurricular forms of work. "The establishment of conditions for an increase in the physical activity of students in their free time is one of the directions that have to be influenced (...) by applying the creativity of the educators in an optimal combination with all possible means and methods" [7]. Mass sports activities in school need more effective scientific and personnel assurance, of development of the science-based programme for agitation and propaganda of physical education and sport among the pedagogical staff, parents and students.

Extracurricular motor activity is a factor for prevention against negative phenomena in society that can be achieved by organizing accessible and age-appropriate forms of physical exercise, sports and tourism. In modern society, the organization of entertainment, fun and different types of recreational services is often called animation, and developers and promoters of animated services receive the status of animators. The essence of animation is to stimulate and satisfy the human needs through direct effects on human`s vital forces. It has a versatile nature, corresponding to the diversity of needs, interests and requirements of the people. According to W. Pompl [8] animation by its very nature represents "stimulation of the body, spirit and social activities of people by people".

Among the various areas of animation is the sport animation that involves participants in active, creative and mutually enriching interpersonal relations through various exercises, games and competitions. The basis of it is the need of movement, expression, social contacts. By clarifying the nature of sports animation, P. Dinev and S. Tomova [9] define it as "a combination of various services necessary to maintain the physical capacity and health status of the users." To this, E. Deliverska [10] adds the conduct of sports training. The more non-traditional and fun is sport animation, the greater the chances are to meet the requirements of the users.

Requirements relating to the qualitative conduct of the activities would not have been accomplished up to standard without the competent and dedicated work of the educator. The professional competence of the educator A. L. Mirzagitova и L. G. Akhmetov [11] define as a conscious activity of the students of the pedagogical specialties aimed at acquiring their own psychology and pedagogical knowledge, improving the pedagogical abilities and the professional qualities.

All said here to reveals that the students of pedagogical disciplines need to develop professional-pedagogical competences to realize sport-animation activity.

\section{METHODOLOGY}

The purpose of the study was to establish the opinion and the attitude of students of pedagogical specialties to the possibility to enrich their general and sports and motor culture and their predisposition to acquire professional-pedagogic competence for realization of sport-animation activity with children of pre-school and school age in an educational environment. The method used was a standardized written inquiry among 85 students from the Faculty of Pedagogy, Trakia University, being trained in the specialties "Preschool and Elementary School Pedagogy" and "Elementary School Pedagogy In A Foreign Language", first, second and third course of EQD "Bachelor" in the summer semester of the academic year 2016/2017. The questions in the inquiry were three with closed scaling answers and two with alternative answers.

\section{RESULTS}

After processing the data from the survey, the following results were obtained. To the question "Do you have any idea what the 
definition of "sporting animation" includes and what are the functions of the sports animator?" $-65,88 \%$ of the inquired students do not have sufficient awareness of the sport animation and
$21,18 \%$ responded rather not, another $9,41 \%$ responded rather yes and only 3,53\% responded positively. The results are presented graphically in Figure 1.

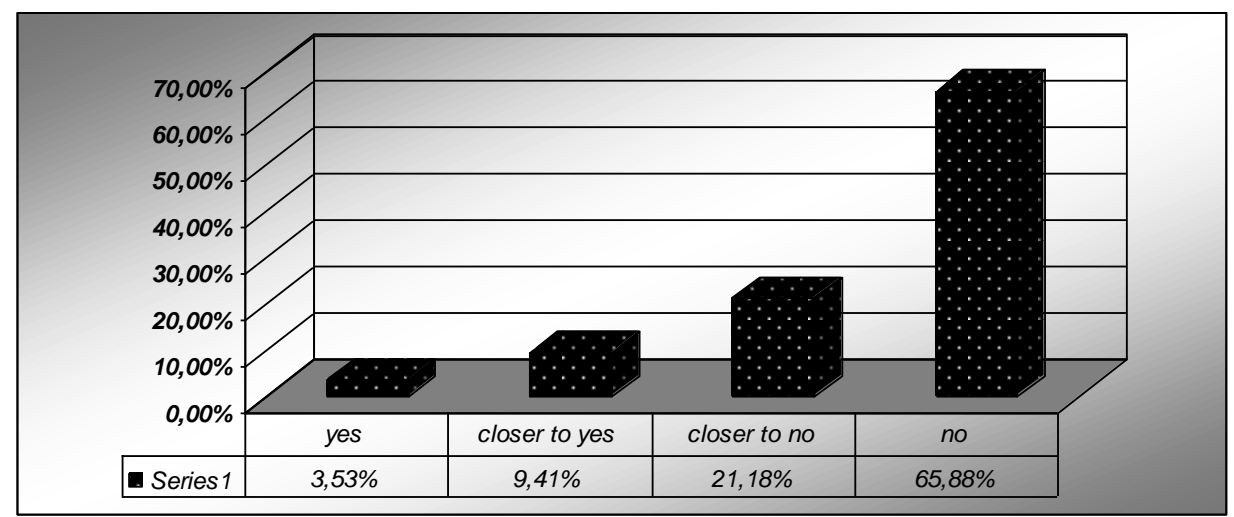

Figure 1. Answer to the question"Do you have any idea what the definition of "sporting animation" includes and what are the functions of the sports animator?"

On the question"Are you ready to prepare a scenario and to organize a sports day or sports competition for children/ students in the educational establishment, at a sea or a mountain camp, tourist excursions and hikes, "green" school, etc.?" - 77,65 \% responded that they had no knowledge, $11,75 \%$ were close to answering no, $8,25 \%$ responded close to yes and only $2,35 \%$ answered positively. The results are presented graphically in Figure 2.

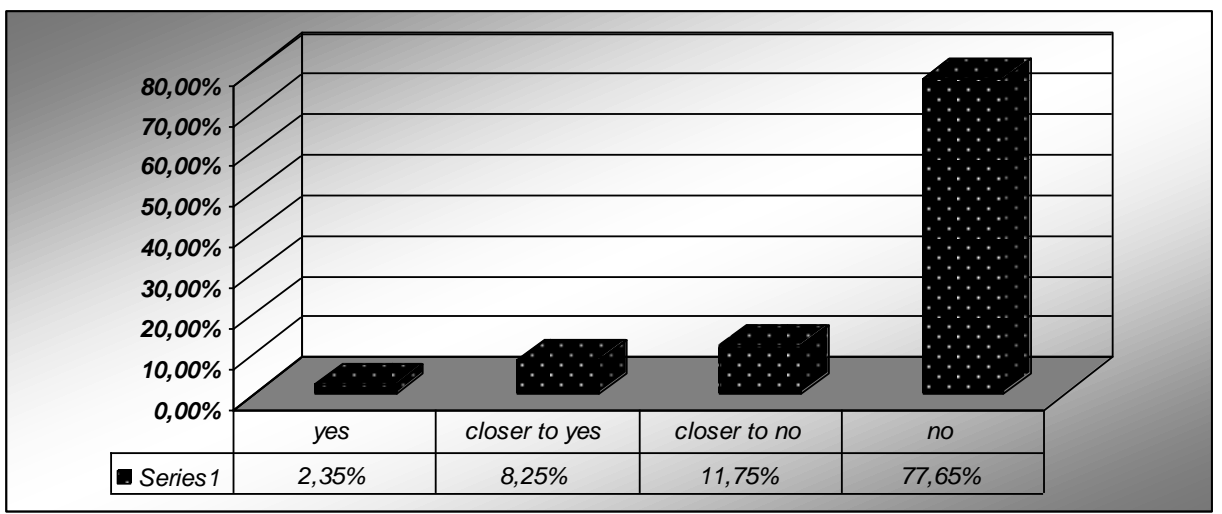

Figure 2. Answer to the question"Are you ready to prepare a scenario and to organize a sports day or sports competition for children/ students in the educational establishment, at a sea or a mountain camp, tourist excursions and hikes, "green" school, etc.?"

To the question "Would you choose "Sport animation in an educational environment" as an elective discipline, if suggested?" - 84,71\% of respondents respond positively, $4,70 \%$ are close to answering yes, $1,18 \%$ responding close to no and $9,41 \%$ responding negatively. The results are presented graphically in Figure 3.

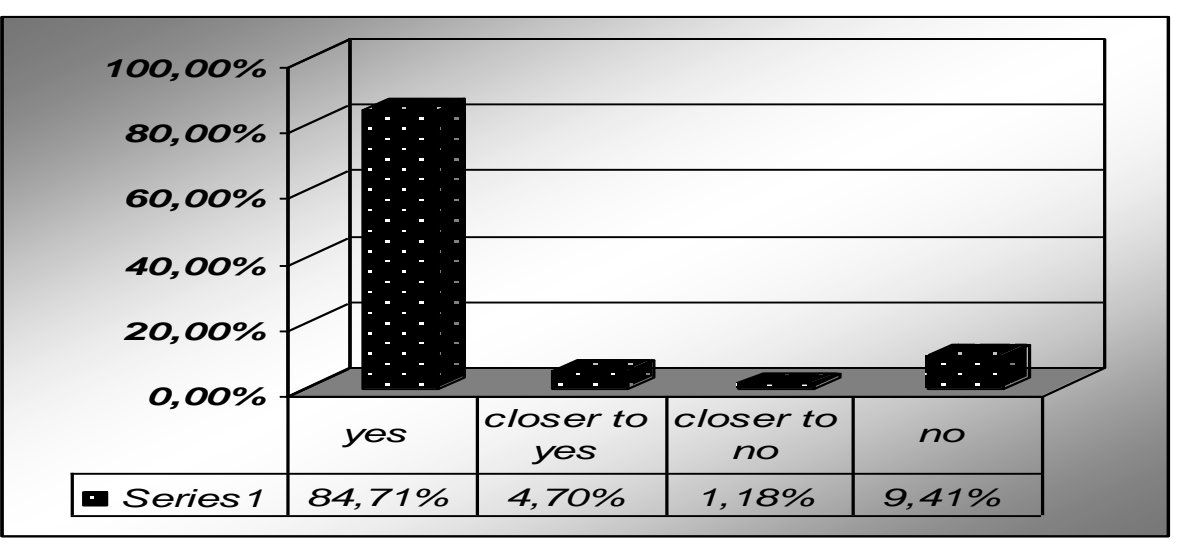

Figure 3. Answer to the question"Would you choose "Sport animation in an educational environment" as an elective discipline, if suggested?" 
On the question "Do you consider that the knowledge, skills and competences you would get from training in the elective discipline "Sport animation in an educational environment", would be useful for your professional realization?" - a sufficiently high percentage $(92.94 \%)$ of respondents answered positively and only $7.06 \%$ responded negatively. The results are presented graphically in Figure 4.

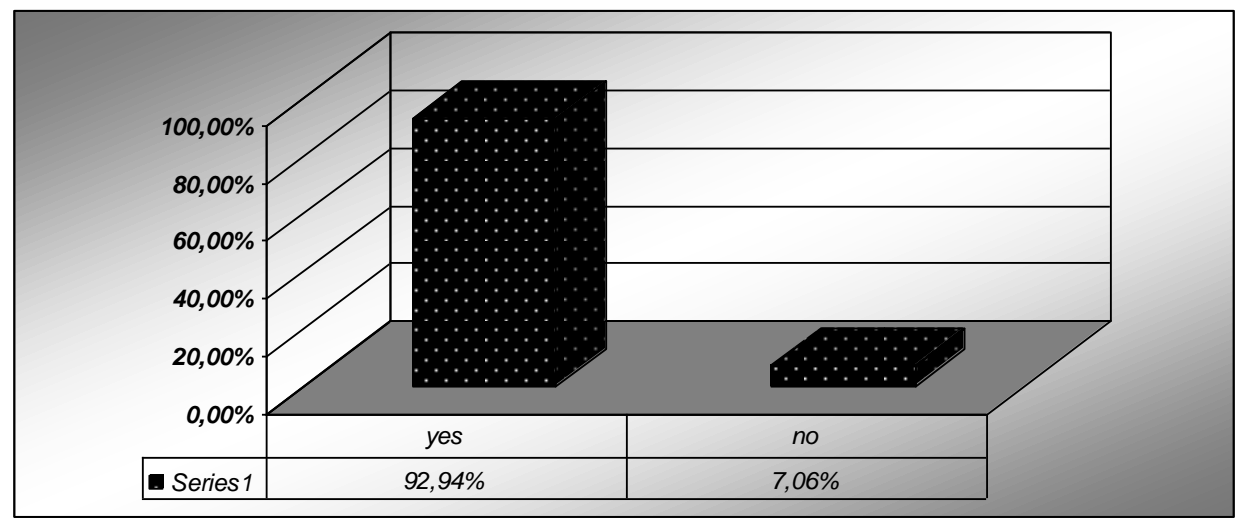

Figure 4. Answer to the question"Do you consider that the knowledge, skills and competences you would get from training in the elective discipline "Sport animation in an educational environment", would be useful for your professional realization?"

To the question "Do you consider that the basic preparation in sport animation would be useful for your personal development and social activity beyond your professional activity of an educator?" - over $90 \%$ of respondents respond positively. The results are presented graphically in Figure 5.

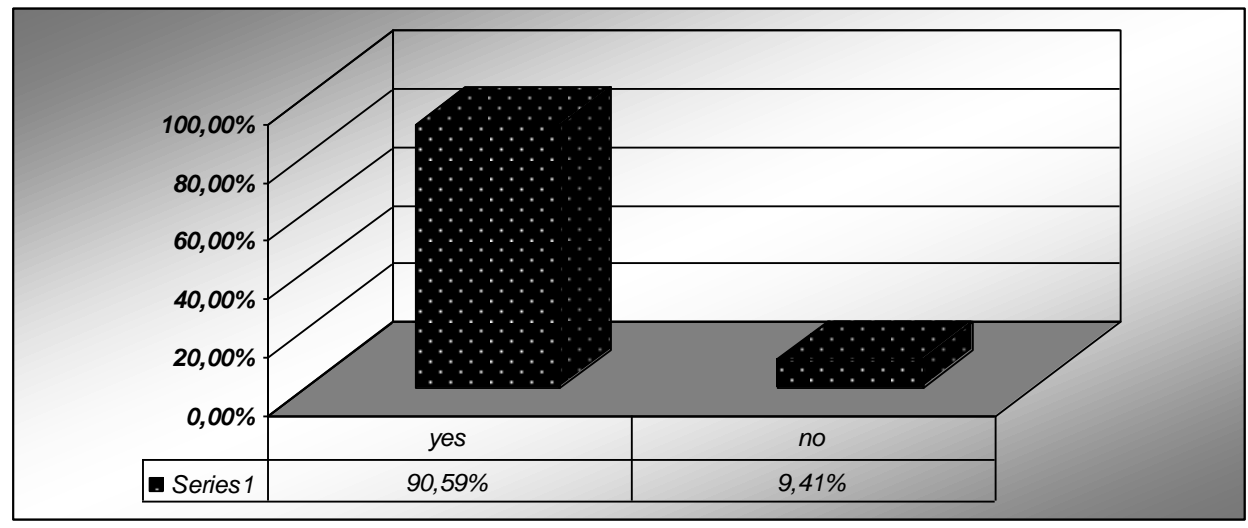

Figure 5. Answer to the question "Do you consider that the basic preparation in sport animation would be useful for your personal development and social activity beyond your professional activity of an educator?"

\section{CONCLUSION}

The analysis of the results shows:

1. Most of the students have no readiness to organize a sports day or sports competition in an institution-organized environment.

2. Students do not have sufficient awareness of the sport animation and want to be trained in this elective discipline.

3. The majority of the students inquired see the benefits of being trained on their professional and pedagogical activities, and beyond.

The updates of the regulatory requirements of the NAEA - the National Agency for the Evaluation and Accreditation of higher education, and the Law on Higher Education as a core paradigm, has set the task of developing and implementing value-oriented reflective technology for organizing of the training process in the system of higher education. In order to meet the requirements of modern society, the educator has to, in particular the teacher, continually strives to improve his/her work. Instead of applying the conventional, the educator has to be creative when solving the tasks set, showing a heightened sense of responsibility and not sparing himself/ herself. I. Shivacheva [12] states that teachers, as representatives of the most widely applicable pedagogical profession, show aspiration not be only a source of information, but to promote the development of competences for their independent applying in different situations. 
Today's dynamic and high technology world sets great challenges to man. It is difficult to prove that activities with physical exercise and sport in childhood prolong human life, but it is certain that they lead to building of habits related to ignoring the hypodinamics and its consequences. Physical activity is extremely important for health, the most important factor for human survival.

\section{REFERENCES}

1. European policy framework and strategy for the 21st century "HEALTH 2020". World Health Organization. www.ncphp.government.bg/files/nczi/Healt h2020_BG [Accessed on 01.02.2018].

2. Convention on the Rights of the Child. www.unicef.bg/assets/CRC_Materials/CRC _bg.pdf [Accessed on 02.02.2018].

3. Merdzhanova, E. Physical activity of the population in Bulgaria. - B: Nursing science, XLVI, 1, 49-51, 2014.

4. Koleva, N. School Hygiene (teaching tool). Sofia: UPH "SV. Kliment Ohridski", 1995.

5. Popivanova, C. Immobilization of adolescents in school age. - B: Sports and Science, 3, 33-45, 2005.
ZHELEVA-TERZIEVA D.

6. Yordanov, S., L. Dimitrov, E. Nikolova. Extracurricular and extra-school activities in Physical Education, Sport and Tourism. B: Sports and Science, 2, 102-107, 2002.

7. Naidenova, K. Status of motor activity in 10-14-year-old schoolgirls. Veliko Turnovo: NMU, 2017.

8. Pompl, W. The concept of animation: Aspects of tourism services. - B: Tourism Management, 4 (1), 3-11, 1983.

9. Dinev, P., S. Tomova. Sport animation. Varna: UE "Science and economics", 2008.

10.Deliverska, E. Competencies and profile of the sports animator. Abstract. Blagoevgrad, 2014.

11.Mirzagitova, A. L., L. G. Akhmetov. SelfDevelopment of Pedagogical Competence of Future Teacher. Russia: Kazan Federal University - International Education Studies, 3, 9020-9039, 2015.

12.Schivacheva-Pineda, I. The creative "specifics" in teachers' professional work, Yambol: Technical College, 2003. 